\title{
A Unified Representation for Morphological, Syntactic, Semantic, and Referential Annotations
}

\author{
Erhard W. Hinrichs, Sandra Kübler, Karin Naumann \\ SfS-CL, University of Tübingen \\ Wilhelmstr. 19 \\ 72074 Tübingen, Germany \\ \{eh, kuebler, knaumann $\}$ esfs. uni-tuebingen. de
}

\begin{abstract}
This paper reports on the SYN-RA (SYNtax-based Reference Annotation) project, an on-going project of annotating German newspaper texts with referential relations. The project has developed an inventory of anaphoric and coreference relations for German in the context of a unified, XML-based annotation scheme for combining morphological, syntactic, semantic, and anaphoric information. The paper discusses how this unified annotation scheme relates to other formats currently discussed in the literature, in particular the annotation graph model of Bird and Liberman (2001) and the pie-in-thesky scheme for semantic annotation.
\end{abstract}

\section{Introduction}

The purpose of this paper is threefold: (i) it discusses an annotation scheme for referential relations for German that is significantly broader in scope than existing schemes for the same task and language and that also goes beyond the inventory of anaphoric relations included in the pie-in-the-sky sample feature structures ${ }^{1}$, (ii) it presents a unified, XML-based annotation scheme for combining morphological, syntactic, semantic, and anaphoric information, and (iii) it discusses how this unified annotation scheme relates to other formats currently discussed in the literature, in particular the annotation

\footnotetext{
${ }^{1}$ See e.g. nlp.cs.nyu.edu/meyers/pie-in-the-sky/ analysis5.
}

graph model of Bird and Liberman (2001) and the pie-in-the-sky scheme for semantic annotation ${ }^{2}$.

\section{Referential Relations}

This section introduces the inventory of referential relations adopted in the SYN-RA project. We define referential relations as a cover-term for all contextually dependent reference relations. The inventory of such relations adopted for SYN-RA is inspired by the annotation scheme first developed in the MATE project (Davies et al., 1998). However, it takes a cautious approach in that it only adopts those referential relations from MATE for which the developers of MATE report a sufficiently high level of interannotator agreement (Poesio et al., 1999).

SYN-RA currently uses the following subset of relations: coreferential, anaphoric, cataphoric, bound, split antecedent, instance, and expletive. The potential markables are definite NPs, personal pronouns, relative, reflexive, and reciprocal pronouns, demonstrative, indefinite and possessive pronouns.

There is a second research effort under way at the European Media Laboratory Heidelberg, which also annotates German text corpora and dialog data with referential relations. Since their corpora are not publicly available, it is difficult to verify their inventory of referential relations. Kouchnir (2003) has used their data and describes the relations anaphoric, coreferential, bridging, and none.

Following van Deemter and Kibble (2000), we define a coreference relation to hold between two

\footnotetext{
${ }^{2}$ See nlp.cs.nyu.edu/meyers/pie-in-the-sky/ pie-in-the-sky-descript.html.
} 
NPs just in case they refer to the same extralinguistic referent in the real world. In the following example, a coreference relation exists between the noun phrases [1] and [2], and an anaphoric relation between the noun phrase [2] and the personal pronoun [3]. Since noun phrases [1] and [2] are coreferential, all three NPs belong to the same coreference chain. In keeping with the MUC-6 annotation standard $^{3}$, we establish the anaphoric relations of a pronoun only to its most recently mentioned antecedent.

(1) 11 Der neue Vorsitzende der Gewerkschaft The new chairman of the union

Erziehung und Wissenschaft] heißt [2 Ulli

Education and Science is called Ulli

Thöne]. [3 Er] wurde gestern mit 217

Thöne. He was yesterday with 217

von 355 Stimmen gewählt.

out of 355 votes elected.

'The new chairman of the union of educators and scholars is called Ulli Thöne. $\mathrm{He}$ was elected yesterday with 217 of 355 votes.'

Cataphoric relations hold between a preceding pronoun and a following antecedent within the same sentence, even if this antecedent has already been mentioned within the preceding text. An example for a cataphoric relation is shown in (2).

(2) Vier Wochen sind [sie] nun schon in Berlin, Four weeks are they now already in Berlin, [die 220 Albaner aus dem Kosovo]. the 220 Albanians from the Kosovo.

'They have already been in Berlin for four weeks, the 200 Albanians from Kosovo.'

The relation bound holds between anaphoric expressions and quantified noun phrases as their antecedents (see example (3)).

(3) [Niemandem] fällt es schwer, das Bild To nobody is it difficult, the picture vor [sich] zu sehen. in front of himself to see.

'Nobody has trouble imagining the picture.'

\footnotetext{
${ }^{3}$ See www.cs.nyu.edu/cs/faculty/grishman/ cotask21.book_1.html.
}

The split antecedent relation holds between coordinate NPs/plural pronouns and pronouns/definite NPs referring to one member of the plural expression. In example (4), the indefinite pronoun beide enters into two split antecedent relations, with noun phrases 1 and 2.

(4) Aber plötzlich gibt es da einen völlig But suddenly gives it there a completely unglaubwürdig und grotesk wirkenden implausible and grotesque seeming Anruf [1 des Detektiven] bei [ 2 der phone call of the detective to the Mutter des Opfers], [beide] weinen mother of the victim , both cry sich minutenlang etwas themselves for some minutes something vor $\quad, \ldots$ verb part , ...

'But suddenly, there is a completely implausible and grotesque phone call from the detective to the mother of the victim, they both cry at each other for several minutes, ...'

An instance relation exists between a preceding/following pronoun and its NP antecedent when the pronoun refers to a particular instantiation of the class identified by the NP.

(5) Die konservativen Kräfte warten ja nur The conservative powers wait just only darauf, ihm [Sätze] um die Ohren zu for that, him sentences around the ears to hauen wie [jenen von den 16 hit like the one about the 16 Mittelstrecklern], denen er in vier middle-distance runners, to whom he in four Wochen die Viererkette weeks the double full-back formation beibringe. teaches.

'The conservative powers are just waiting to bombard him with sentences like the one about the 16 middle-distance runners who he is teaching the double full-back formation in four weeks.' 
In sentence (5), the relation between the two bracketed NPs is an example of such an instance relation since the second NP is a particular instantiation of the referent denoted by the first NP.

A third person singular neuter pronoun es is marked as expletive if it has no proper antecedent. This is the case for presentational es in example (6), impersonal passive as in example (7), or es as subject for verbs without an agent as in example (8).

(6) [1 Es] zeichnet sich die konkrete Möglichkeit It emerges the concrete possibility ab. verb part.

'The concrete possibility emerges.'

(7) [Es] wird bis zum Morgen getanzt. There is until the morning danced.

'People are dancing until morning.'

(8) [Es] steht schlecht um ihn.

It stands bad for him.

'He is in a bad way.'

Apart from expletive uses of es and anaphoric uses with an NP antecedent, the pronoun es can also be used in cases of event anaphora as in sentence (9). Here es refers to the event of Jochen's winning the lottery. Currently, the annotation in SYNRA is restricted to NP anaphora and therefore event anaphors such as in sentence (9) remain unannotated for anaphora.

(9) Jochen hat im Lotto gewonnen. Aber er Jochen has in the lottery won. But he weiss es noch nicht. knows it yet not.

'Jochen has won the lottery. But he does not know it yet.'

The annotation of such relations is performed manually with the annotation tool MMAX (Müller and Strube, 2003). Its graphical user interface allows for easy selection of the relevant markables and the accompanying relation between the contextually dependent expression and its antecedent.

\section{Automatic Extraction of Markables and of Semantic Information}

Annotation of referential relations involves two main tasks: the identification of markables, i.e., identifying the class of expressions that can enter into referential relations, and the identification of the particular referential relations that two or more expressions enter into. Identification of markables requires at least partial syntactic annotation of the text. If referential relations need to be annotated from plain text, then markables must be identified semiautomatically from the output of a chunker or full parser, if available, or otherwise completely manually. However, in each of these two scenarios, identification of markables is a time-consuming process. In case of semi-automatic annotation, the effort required depends on the quality of the parser, but will require at least some amount of manual postcorrection of the parser output.

Identification of markables is considerably easier for treebank data since treebanks already provide the necessary syntactic information. For German, there are currently two large-scale treebanks available: the NEGRA/TIGER (Brants et al., 2002) treebank and the Tübingen treebanks for spoken and written German (Stegmann et al., 2000; Telljohann et al., 2003). All the treebanks were annotated with the help of the annotation tool Annotate (Plaehn, 1998). The treebank annotations are available in the Annotate export format (Brants, 1997) and in an XML format.

The SYN-RA project is based on the Tübingen treebank of written German (TüBa-D/Z). This treebank uses as its data source a collection of articles of the German daily newspaper taz (die tageszeitung). The treebank currently comprises appr. 15000 sentences, with a new release of 7000 additional sentences scheduled for June of this year.

Due to its fine grained syntactic annotation, the TüBa-D/Z treebank data are ideally suited as a basis for the identification of markables and for extracting relevant syntactic and semantic properties for each markable. The TüBa-D/Z annotation scheme distinguishes four levels of syntactic constituency: the lexical level, the phrasal level, the level of topological fields, and the clausal level. The primary ordering principle of a clause is the inventory of topological fields, which characterize the word or- 


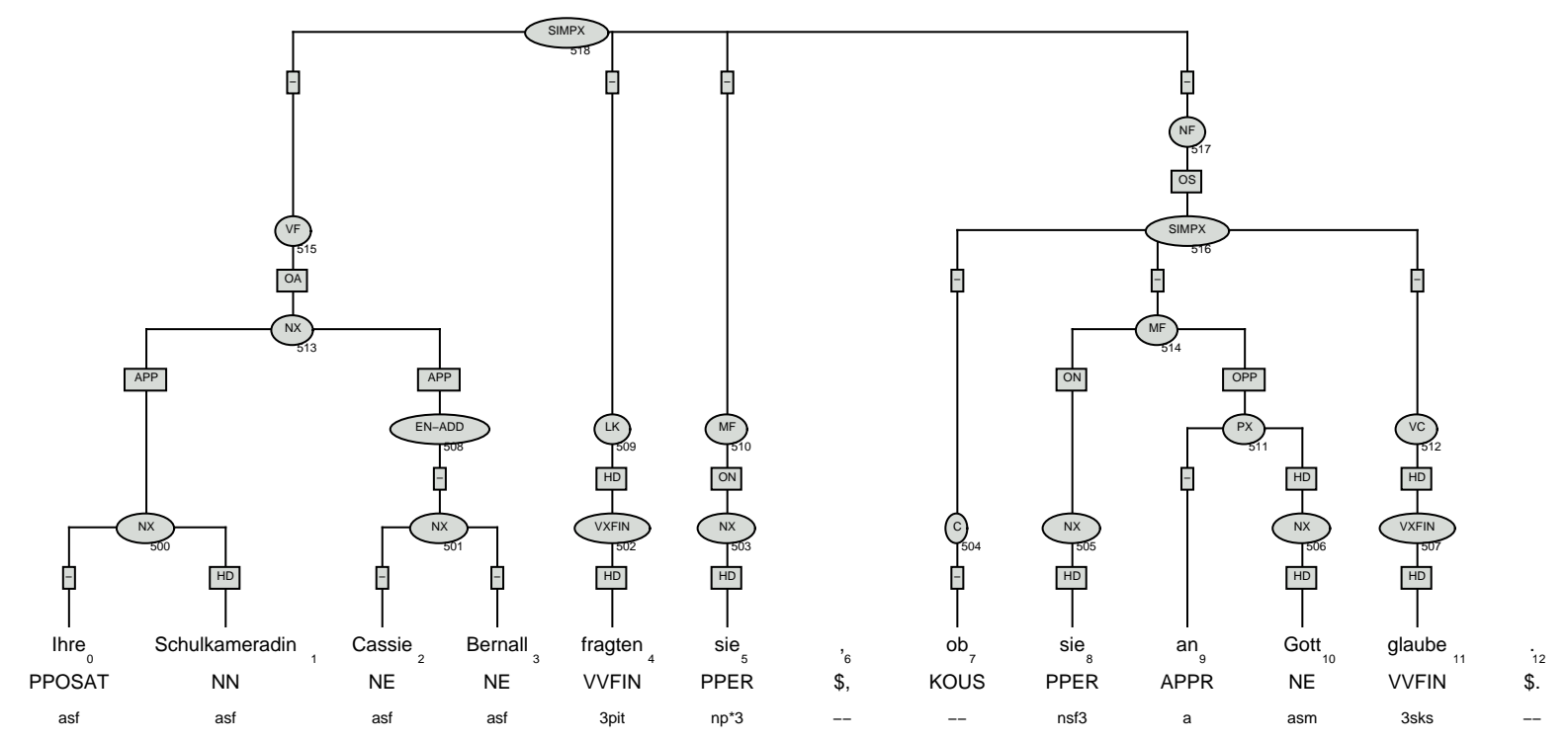

Figure 1: A sample tree from the TüBa/D-Z treebank.

der regularities among different clause types of German and which are widely accepted among descriptive linguists of German (cf. e.g. (Drach, 1937; Höhle, 1986)). The TüBa-D/Z annotation relies on a context-free backbone (i.e. proper trees without crossing branches) of phrase structure combined with edge labels that specify the grammatical function of the phrase in question.

Figure 1 shows an example tree from the TüBa$\mathrm{D} / \mathrm{Z}$ treebank for sentence (10). The sentence is divided into two clauses (SIMPX), and each clause is subdivided into topological fields. The main clause is made up of the following fields: VF (mnemonic for: Vorfeld - 'initial field') contains the sentenceinitial, topicalized constituent. LK (for: linke Satzklammer - 'left sentence bracket') is occupied by the finite verb. MF (for: Mittelfeld - 'middle field') contains adjuncts and complements of the main verb. NF (for: Nachfeld - 'final field') contains extraposed material - in this case an indirect yes/no question. The subordinate clause is again divided into three topological fields: C (for: Komplementierer 'complementizer'), MF, and VC (for: Verbalkomplex - verbal complex). Edge labels are rendered in boxes and indicate grammatical functions. The sentence-initial NX (for: noun phrase) is marked as OA (for: accusative complement), the pronouns sie in the main and subordinate clause as ON (for: nom- inative complement).

(10) Ihre Schulkameradin Cassie Bernall fragten Their fellow student Cassie Bernall asked sie $\quad$ ob sie an Gott they[subj], whether she[subj] in God glaube. believes.

'They asked their fellow student Cassie Bernall whether she believes in God.'

Topological field information and grammatical function information is crucial for anaphora resolution since binding-theory constraints crucially rely on sentence-structure (if the binding theory principles are stated configurationally (Chomsky, 1981)) or on argument-obliqueness (if the binding theory principles are stated in terms of argument structure, as in (Pollard and Sag, 1994)). In the case at hand, the subject pronoun of the main clause, sie, cannot be anaphorically related to the object NP Ihre Schulkameradin Cassie Bernall since they are coarguments of the same verb. However, the possessive pronoun ihre and the subject pronoun sie of the subordinate clause, can be and, in fact, are anaphorically related, since they are not co-arguments of the same verb. This can be directly inferred from the treebank annotation, specifically from the sentence structure and the grammatical function information 
encoded on the edge labels. Most published computational algorithms of anaphora resolution, including (Hobbs, 1978; Lappin and Leass, 1994; Ingria and Stallard, 1989), rely on such binding-constraint filters to minimize the set of potential antecedents for pronouns and reflexives.

As already pointed out, the sample sentence contains four markables: one possessive pronoun Ihre, two occurrences of the pronoun sie and one complex NP Ihre Schulkameradin Cassie Bernall. The latter NP is a good example of SYN-RA's longest-match principle for identifying markables. In case of complex NPs, the entire NP counts as a markable, but so do its subconstituents - in the case at hand, particularly the possessive pronoun ihre. All of this information can be directly derived from the treebank account. Compared to other annotation efforts for German where markables have to be chosen manually (Müller and Strube, 2003), manual annotation in the SYN-RA project can, thus, be restricted to the selection of the appropriate referential relations between referentially dependent expressions and their nominal antecedents.

\section{The Unified, XML-based Annotation Scheme}

The annotation of referential expressions is embedded in a unified format which also contains morphological, syntactic, and semantic information. The annotation scheme is represented in XML, the widely acknowledged standard for exchanging data, which guarantees portability and re-usability of the data. Each sentence, as well as all words and all nodes in the syntactic structure, are assigned a unique ID. These IDs are used in the annotation of referential relations. The annotation of the treebank sentence 11976 (cf. example (10)) is shown in Figure 2.

The sentence number is encoded as the ID of the sentence. The first word, Ihre, has an anaphoric relation to a noun phrase in the previous sentence. This relation is marked in the element anaphora, which gives the antecedent as node 517 of sentence 11975 , i.e. the previous sentence. The other two anaphoric relations are sentence-internal, the first personal pronoun sie having Ihre (id: s11976w0) as antecedent, the second one the noun phrase Ihre Schulfreundin
Cassie Bernall (id: s11976n513). The annotation of the first personal pronoun is an example for the annotation of an anaphoric chain. Ihre and sie belong to the same chain. However, in order to facilitate the extraction of direct relations, such chains are represented in a way that each anaphoric expression refers to the last occurrence of an antecedent.

The SYN-RA scheme is very similar to the MUC-6 coreference annotation scheme ${ }^{4}$ but it is more powerful in two respects: As described above, the inventory is not restricted to coreference and anaphoric relations, it also covers e.g. instance relations or split antecedent relations. The latter relation is also the reason for encoding the relational information as XML elements, and not as attributes of a word or a node. If an anaphor enters into a split antecedent relation, it has more than one distinct antecedent. In this case, the element anaphora has two (or more) relations. Such an example is graphically displayed for sentence (4) in Figure 3. The relevant XML representation of the complex entry for the word beide is shown in Figure 4.

\section{Related Work}

This section discusses how the unified SYN-RA annotation scheme relates to other formats currently discussed in the literature, in particular the pie-inthe-sky scheme for semantic annotation ${ }^{5}$ and the annotation graph model of (Bird and Liberman, 2001). While these two annotation schemes are by no means the only contenders for corpus annotation standards in the literature, they are certainly among the most ambitious and promising.

While the pie-in-the-sky scheme is clearly still under development, the following characteristics and goals can already be gleaned from its webpage and the annotation examples presented there: The annotation is feature-structure-based and incorporates various levels of linguistic annotation, in particular a PROPBANK style predicate-argument structure, dependency style syntactic information, as well as morpho-syntactic and word class information. All this information is rooted in the attributes needed for predicate-argument assignment,

\footnotetext{
${ }^{4}$ See www.cs.nyu.edu/cs/faculty/grishman/ Cotask21.book_1.html.

${ }^{5}$ See nlp.cs.nyu.edu/meyers/pie-in-the-sky/ pie-in-the-sky-descript.html.
} 


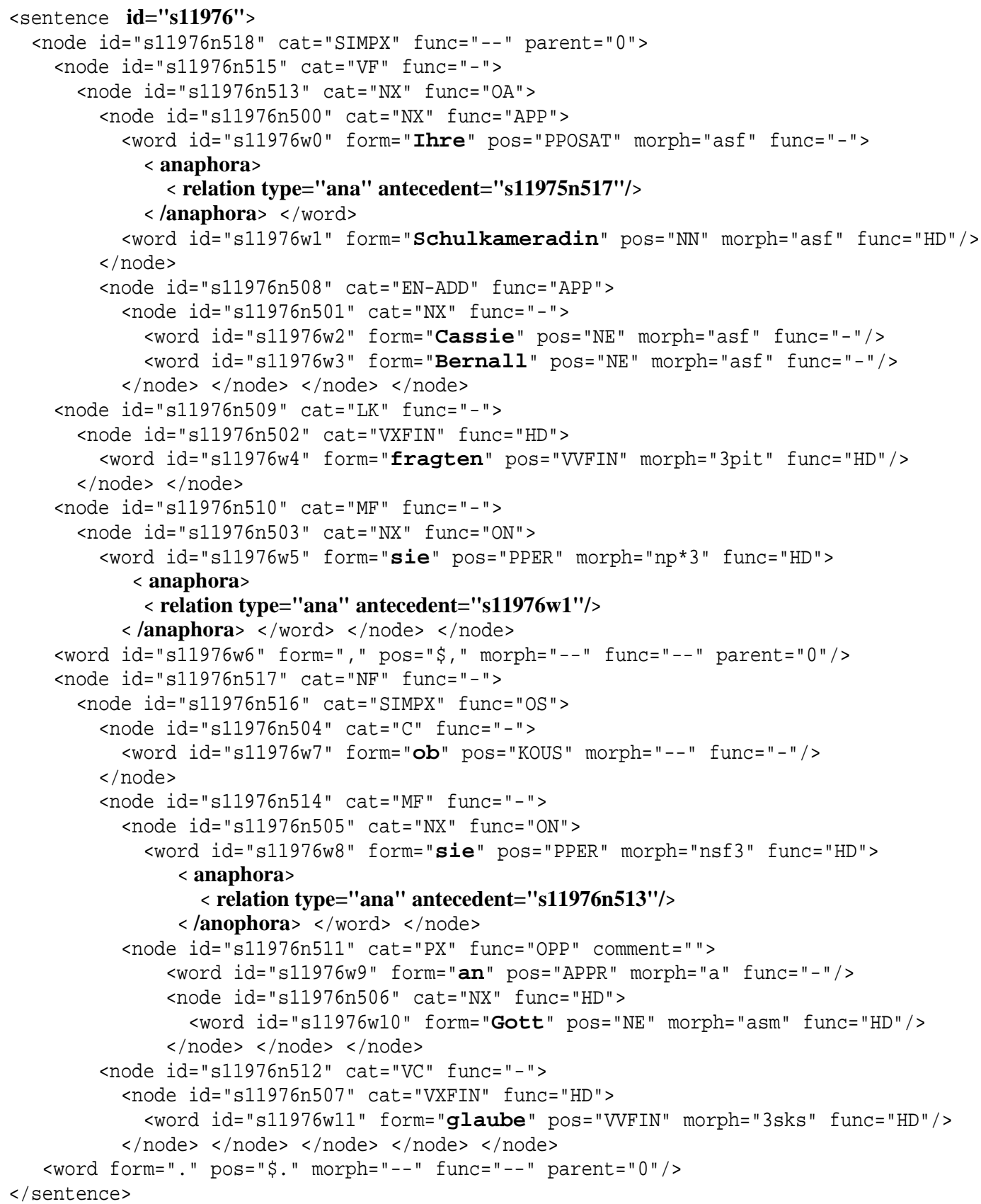

Figure 2: The XML format represents information on all levels of annotation. The words of the sentence and the anaphoric annotation are shown in bold. 


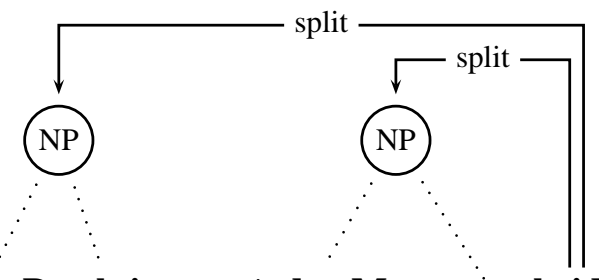

Aber plötzlich gibt es da einen ... Anruf des Detektiven bei der Mutter ..., beide weinen sich minutenlang etwas vor...

Figure 3: The annotation of the split antecedent relation in sentence (4). For representational reasons, the sentence is shortened and only relevant information is displayed. Syntactic boundaries are shown as dotted lines, anaphoric relations as black lines.

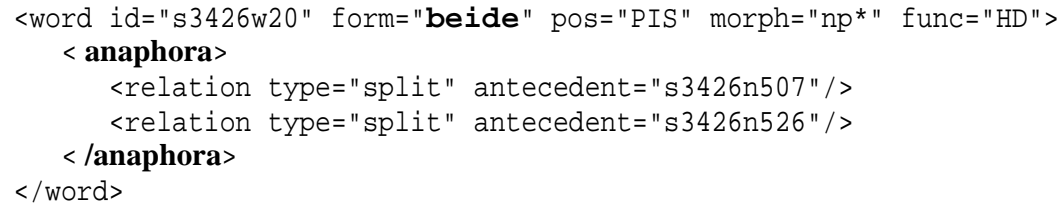

Figure 4: The XML representation of the encoding of split antecedents for the word beide in sentence (4). A graphical representation of the relation is shown in Figure 3. The antecedent "s3426n507" refers to the first NP, "s3426n526" to the second one in Figure 3.

with syntactic and morpho-syntactic information distributed among the corresponding elements in the predicate-argument structure representation. Accordingly, semantic representations provide the organizing principle while morpho-syntactic and syntactic information play a subordinated role.

The SYN-RA annotation scheme resembles the pie-in-the-sky scheme in that it also uses one level of representation, in this case hierarchical syntactic structure, as the organizing principle and treats referential relations, grammatical function information, and morpho-syntactic annotation as subordinated types of information. More generally, the piein-the-sky and the SYN-RA representations offer a particular view of the annotation, each with its own "perspective": semantics-based (pie-in-the-sky) and syntax-based (SYN-RA).

By contrast, Bird and Liberman's (2001) annotation graphs are intended as a graph-based, multilayered annotation scheme where each level of linguistic annotation is treated equally, as an independent layer. The graph-based annotation model is powerful enough to also allow groupings of discontinuous constituents and other non-adjacent linguis- tic phenomena, without having to rearrange the linear order of the input. In both respects, their annotation model is maximally general.

\section{Future Directions}

In the previous section we have compared two perspective-dependent annotation schemes that use a particular level of linguistic annotation as their primary organizing principle and have contrasted them with the perspective-independent annotation-graph model. We believe that both types of representation models have their independent justification. Perspective-based representations, such as SYNRA and pie-in-the-sky, are well-justified for particular application scenarios. For example, for text summarization and other semantic tasks, the piein-the-sky model seems particularly well-motivated since the pertinent semantic information can be easily extracted from its predicate-argument-structurerooted feature structures. For other tasks, such as anaphora resolution, for which syntactic information is more relevant, the syntax-based representation of SYN-RA allows for an easier extraction of the relevant information for rule-based, statistical, 
and machine-learning approaches to computational anaphora resolution. More generally, perspectivebased representations are highly task-dependent. It would be misguided to consider them as ideal, taskindependent annotation standards. If one wants to establish a task-independent annotation standard, then a perspective-independent annotation scheme such as the annotation graph model looks like a promising direction for future research. In particular, such research should focus on techniques that allow for easy conversion of perspective-independent representations to task-dependent views of the relevant linguistic information.

\section{References}

Steven Bird and Mark Liberman. 2001. A formal framework for linguistic annotation. Speech Communication, 33(1,2):23-60.

Sabine Brants, Stefanie Dipper, Silvia Hansen, Wolfgang Lezius, and George Smith. 2002. The TIGER treebank. In Erhard Hinrichs and Kiril Simov, editors, Proceedings of the First Workshop on Treebanks and Linguistic Theories (TLT 2002), pages 24-41, Sozopol, Bulgaria.

Thorsten Brants, 1997. The NeGra Export Format for Annotated Corpora. Universität des Saarlandes, Computational Linguistics, Saarbrücken, Germany.

Noam Chomsky. 1981. Lectures on Government and Binding. Foris, Dordrecht.

Sarah Davies, Massimo Poesio, Florence Bruneseaux, and Laurent Romary, 1998. Annotating Coreference in Dialogues: Proposal for a Scheme for MATE. MATE.

Kees van Deemter and Rodger Kibble. 2000. On coreferring: Coreference in MUC and related annotation schemes. Computational Linguistics, 26(2):629-637.

Erich Drach. 1937. Grundgedanken der Deutschen Satzlehre. Diesterweg, Frankfurt/M.

Jerry R. Hobbs. 1978. Resolving pronoun references. Lingua, 44:311-338.

Tilman Höhle. 1986. Der Begriff "Mittelfeld", Anmerkungen über die Theorie der topologischen Felder. In Akten des Siebten Internationalen Germanistenkongresses 1985, pages 329-340, Göttingen, Germany.

Robert J. P. Ingria and David Stallard. 1989. A computational mechanism for pronominal reference. In Proceedings of the 27th Conference of the Association for Computational Linguistics, pages 262-271, Vancouver, Canada.
Beata Kouchnir. 2003. A machine learning approach to German pronoun resolution. Master's thesis, School of Informatics, University of Edinburgh.

Shalom Lappin and Herbert Leass. 1994. An algorithm for pronominal anaphora resolution. Computational Linguistics, 20(4):535-561.

Christoph Müller and Michael Strube. 2003. Multi-level annotation in MMAX. In Proceedings of the 4th SIGdial Workshop on Discourse and Dialogue, Sapporo, Japan.

Oliver Plaehn, 1998. Annotate Bedienungsanleitung. Universität des Saarlandes, Sonderforschungsbereich 378, Projekt C3, Saarbrücken, Germany, April.

Massimo Poesio, Florence Bruneseaux, and Laurent Romary. 1999. The MATE meta-scheme for coreference in dialogues in multiple languages. In Proceedings of the ACL Workshop on Standards for Discourse Tagging, pages $65-74$

Carl Pollard and Ivan Sag. 1994. Head-Driven Phrase Structure Grammar. Studies in Contemporary Linguistics. University of Chicago Press, Chicago, IL.

Rosmary Stegmann, Heike Telljohann, and Erhard W. Hinrichs. 2000. Stylebook for the German Treebank in VERBMOBIL. Technical Report 239, Verbmobil.

Heike Telljohann, Erhard W. Hinrichs, and Sandra Kübler, 2003. Stylebook for the Tübingen Treebank of Written German (TüBa-D/Z). Seminar für Sprachwissenschaft, Universität Tübingen, Tübingen, Germany. 